\title{
A Critique of Methodological Dualism in Education
}

\author{
Jeong A Yang, Jae-Bong Yoo* \\ Department of Education, Sungkyunkwan University, Seoul, Korea \\ *Corresponding Author: yoojb@skku.edu
}

Copyright $\bigcirc 2018$ by authors, all rights reserved. Authors agree that this article remains permanently open access under the terms of the Creative Commons Attribution License 4.0 International License

\begin{abstract}
This paper aims to critically examine the paradigm of methodological dualism and explore whether methodologies in social science currently are appropriate for educational research. There are two primary methodologies for studying education: quantitative and qualitative methods. This is what we mean by 'methodological dualism'. Is methodological dualism appropriate for educational research? The answer is no. When we face new situations in a multicultural society, various types of research can be used according to nature of research. Quantitative and qualitative methods can be virtually integrated. The integration of quantitative and qualitative research can be achieved in the following ways: pragmatic integration, phenomenological integration, and integration in educational practice. We insist that integration in educational practice is the most viable way among the methods of methodological integration.
\end{abstract}

Keywords Methodological Dualism, Integration of Methodologies, Quantitative and Qualitative Methods

\section{Introduction}

Given the rapid pace of globalization, nowadays, many countries face the challenge of educating immigrants and their children. This reality has led to increased opportunities for research into education, enabling studies that use different approaches and discourses. Such variety has led to the enrichment of education research; however, researchers tend to have conducted studies via non-standardized methods. One reason most approaches to education have not achieved unity or consistency is the prevailing "methodological dualism," where studies are performed based on one of two distinct methodologies in education: either quantitative or qualitative research. Is methodological dualism appropriate for educational research? This paper critically examines the methodological dualism of quantitative and qualitative research, discusses three methods of integration and suggests the possibility of integration as a practice in education.

\section{Philosophical Backgrounds in Quantitative and Qualitative Research}

Presuming human life of the natural world, quantitative researchers tend to adopt the methodologies of natural science; they establish a hypothesis, prove the hypothesis, and deduce general laws based on their findings.

Modern natural science is based on philosophical empiricism - the belief that legal knowledge comes from experience alone: reliable knowledge is acquired through the five senses and tested through observations and experiments. Scientific laws are based on observable phenomena that occur repeatedly. These scientific explanations are called "comprehensive model" laws. In natural sciences, explanation and prediction have the same logic, and objectivity relies on a clear division between facts and value judgments. Quantitative research is based on experience, which uses extensive research data, quantification, and statistical analysis to analyze social phenomena and human beings [1].

In quantitative research, the differentiation between physical and social phenomena is unclear, and quantitative researchers believe social phenomena can be interpreted using scientific structures. Thus, educational events can be explained through quantitative descriptions.

However, quantitative research methods have at least two limitations. First, some argue that research methods adapted to natural or physical phenomena cannot be utilized efficiently to understand human society. Human beings have free will and are therefore subject to the law of values, rather than the natural law. For this reason, some argue that research in natural sciences is not the same as research in social sciences, and research methods should differ according to the field of study. Second, the laws of science cannot often be seemed as objective, as human interpretation is inevitably involved. The development of all theories, including scientific theories, requires creative and speculative thinking, which includes subjective 
interpretation. Therefore, the claim that the results of quantitative research are objective can be disputable [1] [11].

Contrary to the quantitative method, qualitative research embraces various methods: reconstructive assessment, ethnography, and so on [5]. In qualitative research, human beings are embedded in society, unceasingly interacting with the world. Human knowledge is understood to be subjective - that is, based on specific circumstances - and formed by interactions between humans. The meaning that we give to an object is constructed by the views of the community rather than the individual.

Since we live in a subjective world created by the thoughts and intentions of social actors, qualitative researchers attempt to discover the intentions, motives, and individual circumstantial interpretations of their research subjects. Qualitative researchers claim that we have inherited specific cultures and languages and such language is used as a social tool to give our subjective senses an objective representation. By using language, we can describe and reconstruct the social world objectively [11].

In qualitative research, education and training start from reality and is investigated through the process of understanding and interpretation [7]. Although language is a social tool in most qualitative research, it is difficult to prove objectivity. In qualitative research, objective reality is not assumed; instead, a "consensus" reality or the "interpreted" reality is considered. Therefore, the realities found in qualitative research vary according to the researcher's interpretation; in this respect, the qualitative method has less objectivity. Thus, it is challenging to develop standard rules and patterns, and suggest a consistent policy direction. For example, when children from minority cultural backgrounds are shown to have lower average test scores than those from the dominant culture, different interpretations are made. Some researchers may argue that the reason for the students' low academic achievement is their lack of understanding of the language used in the classroom because of their different cultural background. Therefore, they argue that local language education should be strengthened. In contrast, others may believe that their low academic performance is due to their economic condition rather than cultural background and suggest economic equality to resolve the problems arising in intercultural education. Furthermore, some researchers may argue that the form of a test cannot measure academic achievement, so the results cannot be trusted. Thus, at the national level many policy makers are confused and do not believe the results of qualitative research. Consequently, they need consistent solutions for educational problems at the national level.

At first sight, quantitative and qualitative research are quite different. Those who engage in qualitative research attempt to understand the human state using an interpretative or constructivist approach rather than an objective or positivist approach. Unlike qualitative researchers, quantitative researchers naturally tend to see the findings of qualitative research with skepticism because of the absence of methodological objectivity.

However, is methodological dualism correct? In epistemology, education research cannot be defined by only one epistemic viewpoint. Education includes both objective and subjective perspectives. Regarding ontology, it is wrong to assume that quantitative researchers are realists and qualitative researchers are not. Any researcher can use any research method regardless of methodology. Quantitative and qualitative research can be logically divided; however, they are integrated into reality.

\section{Pragmatic and Phenomenological Integration in Education}

If qualitative and quantitative methods are virtually integrated, how can we integrate the two methodologies? There are three ways to integrate them: pragmatic integration, phenomenological integration, and integration as a practice.

First, pragmatic integration, which is the most common way of integration, focuses on the usability or utility of research. The focus is on how well the research describes various phenomena beyond the dualistic thinking that creates a divide between quantitative and qualitative research. Pragmatic researchers can select an appropriate research method to describe phenomena. In other words, researchers can mix quantitative and qualitative methods, along with conceptual analysis and hermeneutical methods. This approach is the "mixed study" (mixed research) method. The current meaning of this expression is determined by the practical results derived from "using of faith and expression." Pragmatic and pluralistic research methods enable communication between quantitative and qualitative research [5].

However, pragmatic mixed studies are limited by their implicit refusal to accept the epistemological characteristics of quantitative and qualitative research. Mixing quantitative and qualitative research can result in potential distortion due to the intentions of a researcher, because this type of integration may only occur for practical purposes. In short, although pragmatic studies facilitate dialogue between research methods, studies using mixed research methods cannot efficiently perform both functions of quantitative and qualitative research.

Assume that there is a study on multicultural students' school maladjustment. Researchers may study this problem using mixed research methods. They can identify the number of maladjusted students, establish a hypothesis, and verify it. Thus, both quantitative and qualitative methods can be used to test this hypothesis. For example, the researchers who have previously engaged in quantitative research may try to carry out qualitative 
research on the philosophical basis of the quantitative research. When conducting qualitative research, the researcher should not assume that the researcher is value-neutral. Researchers should proceed with the assumption that they are humans with prejudice. However, researchers who are used to carrying out quantitative research regard themselves as value-neutral, generating distortions due to their values in qualitative research. Finally, qualitative research is performed only in a manner in helping quantitative research.

Second, phenomenological integration leads to the integration of quantitative and qualitative research based on phenomenological epistemology. From a phenomenological perspective, the difference between quantitative and qualitative research lies not only in a difference in perception but differing interpretations of a specific phenomenon [9]. When we study social phenomena, whether a qualitative or quantitative research method is chosen depends on the attitude of the researcher. Like two sides of a coin, quantitative and qualitative research reflects opposite sides of the experience.

People experience differently due to their individual perspectives and attitudes. Similarly, researchers can interpret the same experience in a variety of ways. For instance, suppose that there is a class of students from different cultural backgrounds. The phenomenon that 'students from different cultural backgrounds exist' is acknowledged by quantitative and qualitative researchers. However, quantitative researchers focus on how many students come from different cultural backgrounds, where these students are from, and what scores they achieve, whereas qualitative researchers concentrate on how students who belong to specific groups feel and think about the situation, how the teachers interpret the situation, how minority students express their thoughts, and so on. Thus, quantitative and qualitative methods result in different interpretations of the same phenomenon according to their focuses and attitudes. The phenomenological integrated approach integrates the two methodologies in phenomenology.

This integration seems to be sound, but it also has some limitations. It attempts to integrate the two methodologies in qualitative research; thus, quantitative research is relatively disregarded. All facts, including those derived from quantitative research, are merely interpretations. However, quantitative researchers aim to reveal the objective reality and believe what they discover should not base on interpretations, but objective facts. They believe that the universe has discernable patterns and rules which researchers should try to find. As a result, quantitative researchers argue that theories and interpretations should be revised whenever researchers find new evidence. Although researchers cannot be objective, they should pursue objectivity in a strict sense. Interpretations cannot be accepted without proven facts. Thus, quantitative researchers cannot accept the assumptions of phenomenological integration. For example, the fact that many minorities have trouble achieving good grades in South Korea is a fact rather than an interpretation.

\section{Integration as a Practice in Education}

Integration as a practice in education aims to integrate the quantitative and qualitative methods regarding praxis. Integration as a practice tends to view education as praxis [2]. Both methodologies play a role in social science. Social science aims to examine society and suggest sound theories. However, educational research should not only examine educational situations and establish theories, but also maintain educational values and suggest solutions for educational problems. Education has always been a praxis for extrinsic and intrinsic purposes. Educational research should perform to achieve both the extrinsic and intrinsic purposes of education within "educational practice."

What is educational research? How is it different from education research? Pring distinguishes "research which is firmly embedded within the social sciences" from "research which many well be relevant to education and research which arises from distinctively educational concerns and which draws upon, but it is not to be reduced to, the knowledge which has accumulated within those science" [11] (Pring, 2000, p.22). The former is called "education research" and the latter "educational research." Educational research is a study that has characteristics that can be said to be educational and related to activities performed by teachers. On the other hand, educational research can be utilized to study various subjects, but should ultimately contribute to the understanding and improvement of educational practice. Furthermore, the interests of the research should be educational. The methodology of educational research should be flexible according to the interest in educational practice. Despite the clarity of objectivity, the methodology based on social sciences research and its pursuit of academic rigor, not all researches seen as educational researches. The academic precision of social science methods and research method models are often inadequate in exploring the values and complex interactions with educational practice [12].

What is an "educational practice"? To understand this concept, it is necessary to understand Alasdair MacIntyre's concept of "practice." According to MacIntyre, people have no absolute or ultimate purpose, but their purpose is formed and revealed by narrative of a human's life, practices, and traditions. The sociality and community of virtue can be better secured by social activities and traditions that people have engaged in and participated in until now. A practice cannot help but be a social practice in that it has a social nature and cannot be understood separate from social context [13]. He defines the practice as follows: 
Any coherent and complex form of socially established cooperative human activity through which goods internal to that form of activity are realized in trying to achieve those standards of excellence in the course which are appropriate to, and partially definitive of that form of activity, with the result that human powers to achieve excellence, and human conceptions to the ends and goods involved, are systematically extended [8] (MacIntyre, 1984, p. 187).

According to this definition, the concept of practice has the following traits. First, a practice includes complex human activities in society and is fundamentally related to human activity. A practice refers to a coherent form of socially established cooperative human activities. Second, a practice concerns the achievement of internal goods. Goods internal to a practice 'can only specify them' in terms of the specific kind of activity and can only be identified and recognized by the experience of participating in the practice in question. Third, a practice has standards of excellence, which activities should achieve. The standards of excellence can vary, depending on each practice. Fourth, a practice consists of rule-governed of rule-following activities. Finally, a practice itself is improved through the ongoing debates of practitioners who engaged in the practice [8] [13].

Wilfred Carr describes what "educational practice" is. Carr asserts that the meaning of "practice (praxis)" that we currently use is inaccurate. When we claim that "practice" is the opposite of theory, we have misunderstood "practice." We often regard practices as everything except theory. This usage of practice is inappropriate in the education field. For instance, we commonly assume that practice is "doing something specific" while a theory is "knowing something universal." However, when we decide what to teach, we should consider not only "knowing" but also "doing." In the real world, educational situations are very complicated and involve both doing and knowing. In short, "educational practice" is not the opposite of theory; instead, it is another concept that should be used to understand education [7].

Carr suggests that we should think about the Greek concept of praxis because in doing so, we can avoid modern prejudices [3] [4]. Aristotle introduced three concepts: theoria (theory), praxis (practice), and poiesis (production). Theoria relates to theoretical activities, praxis relates to communal life, and poiesis relates to activities performed to produce things. Each activity involves intellectual devotion and moral considerations. Theoria, praxis, and poiesis require knowledge or reasoning: epistemme, phronesis, and techne, respectively.

In other words, theoria/ praxis/ poiesis correspond to ways of life, while epistèmè/ phronesis/ techne correspond to knowledge and reasoning. This classification of knowledge compels us to reconsider the definition of education. Carr suggests that the difference between praxis and poiesis is more important than the difference between theoria and praxis. Poiesis aims value neutrality, while praxis aims to pursue value. The value of praxis is realized through practicing activities. If praxis focuses on the results or fails to pursue value, it becomes poiesis [12].

Improving praxis may depend on improving the phronesis of those who practice [12]. What does it mean to view education as praxis? What does praxis mean? Education refers to the activities that lead to learning. Therefore education research focuses on learning. For these reasons, the center of learning may form the cognitive discernment that Peters mentions [11]. Education in specific terms is a learning activity that is considered valuable. Whether certain knowledge is considered valuable is influenced by social culture. Even though informal education by itself cannot educate younger generations, it is necessary to consider it in developing curricula [11]. After all, education involves intentional teaching and directed learning that introduces students to the public world. Teaching is a social practice in which individuals share and participate. Teaching relies on the process of mediation to convey public knowledge, ideas and criticism in the form of personal questions to young people [11]. The process aims to inculcate students with a solid understanding of the curriculum so that they can use propositional knowledge [11]. Therefore, education research should also be concerned with the interactions between teachers and students. It should focus on knowledge, criticism, teaching and learning contents for different types of people initiated into the world of public value, including the different levels of learning activities. Additionally, educational research should contribute to human growth [11]. If this is the case, should research on educational practice follow a specific method? Unlike the metaphysical, educational practice is in the physical world. Education research and evaluation should be done in a practical area, based on the relevance of educational value created during the interaction of teaching and learning. The dualism (separation) of quantitative research and qualitative research in terms of praxis is due to misinterpretation of the nature and complexity of what is being researched. In the light of the nature of "educational" research, rather than research "on education," both methodologies should be used to complement one another [11]. In figurative terms, quantitative study provides general information about the forest, while qualitative research provides specific information about individual trees, and when the two methods are combined, we may fully understand the condition of the entire forest and the individual trees of which the forest is composed. For example, suppose that there is a city with a secondary school dropout rate. A study regarding what happens in the classroom where the dropout rate is high in the city may only be examined through qualitative research. Qualitative research can be conducted by interviewing students who have dropped out, finding out what happened in schools and reasons why they dropped out. At the same time, 
through interviews researchers can obtain teachers' perspectives on what happened. By studying the current educational situation in the classroom, researchers can identify the problematic situation. Such investigation may reveal to us that "students are unable to follow the class because of its overly difficult curriculum." Furthermore, quantitative research can be used to determine if these results are applicable to the whole city. Quantitative researchers set a hypothesis based on the results and conduct a study to test this hypothesis. If a researcher sets the hypothesis as "dropouts occur due to an overly difficult curriculum," they will design a study to investigate what kind of curriculum is difficult, what kind of curriculum influences students, and whether the influence of the curriculum is related to dropouts or not. If the results revealed to be true within the error range, in qualitative research, it is verified through various qualitative methods such as ethnography and in-depth interview. In sum, both quantitative research and qualitative research can help discover ways to better implement education. Thus, the distinction between quantitative and qualitative methodologies in social sciences comes from the error of modern epistemology, which distinguishes between physical and mental, objective and subjective, and external and internal worlds. This division is not based on the validity of the task but philosophical epistemology. Educational research belongs to both areas and methodologies at the same time. "Educational research" can take a variety of approaches depending on the nature of the specific subject being dealt with.

Both methods only recognize one aspect and capture the complexity and diversity of educational practice, the common world in which this praxis exists. Educational research accepts this and is interested in understanding and improving educational practice. In other words, it is interested in various conditions of interactions related to the teaching and learning, and the quality of the learner's experience of human growth. Educational research can help develop problem awareness and define research problems through the research results of both approaches. However, it is necessary to develop a theoretical framework that can bridge the existing boundaries and link "praxis" [11].

\section{Conclusions}

In this paper, we have critically examined the dualism of quantitative methods and qualitative methods and explored the possibilities for integration. Quantitative research and qualitative research seem to be fundamentally different. Quantitative research is based on empirical epistemology, which prefers experimental studies and surveys in developing objective and empirical knowledge. In contrast, qualitative research is based on constructivist epistemology, which explores daily living skills and phenomenological research to interpret phenomena and derive meaning. Given their different epistemologies, quantitative research and qualitative research are logically separate but are actually inseparable. It is perhaps natural to explore a variety of integration methods in educational research regarding this topic. Each of the three integrated approaches proposed — pragmatic integration, phenomenological integration, and integration as a practice - have both advantages and disadvantages. Pragmatic integration integrates the two approaches by attempting to facilitate communication between the two methods, but it also prone to potential distortions of quantitative research and qualitative research, according to the intention of the researcher. The phenomenological integration of quantitative research and qualitative research claims that experiences are viewed differently depending on the perspective through which the experience is observed and the attitude of the observer. However, because of the theories underlying phenomenology, whether such research can pursue the goal of quantitative research may be called into question. Integration as a practice is based on the belief that education is praxis and tries to integrate quantitative and qualitative research in praxis. An educational practice aims to facilitate the participation of young people in society, including traditional activities that mediate knowledge and critical thinking. Research on how to enhance this function is called "educational research" in praxis. Educational research focuses on how the interaction between teaching and learning can best be performed.

Educational philosophy as an educational practice aims to provide a new orientation for education. Studies on education have a complexity that cannot be resolved through methodological dualism. Therefore, research on education should examine a variety of interactions between students and educators rather than exclusively embrace methodologies based on the dualism of either quantitative research or qualitative research. Education researchers should concentrate on the interactions between teachers and students, take into consideration the specific circumstances in which education takes place, reveal the processes of education, and integrate other suggestions and practical methods to achieve desired educational outcomes. In the dynamic environment of the classroom, teachers will face many situations that cannot be resolved solely by the theories that guide traditional customs and curricula. Educators and teachers should try to address the problems within specific situations that arise in praxis rather than embrace theories based on dualism.

\section{REFERENCES}

[1] Benton, T. and Craib, I. Philosophy of Social Science, New York: Palgrave, 2001. 
[2] Carr, W. What is an Educational Practice?, Journal of Philosophy of Education, Vol. 21, No. 2, 163-175, 1987.

[3] Carr, W. The Gap between Theory and Practice. For Education: Toward Critical Educational Inquiry, Buckingham: Open University Press, 1995.

[4] Carr, W. What is an Educational Practice for Education: Toward Critical Educational Inquiry, Buckingham: Open University Press, 1995.

[5] Cho, Y.D. Qualitative Research Methodology: Theory [In Korean]. Seoul: Dreampig, 2015.

[6] Dilthey, W. Ausgewahlte Schriften zur Padagogik [Collection of Pedagogy (translated by Song S.N in Korean)]. Seoul: Jimanji, 2008.

[7] Kwak, D.J., Na, B.H. and Yoo, J.B. Doing Philosophy of Education as 'Practical Philosophy': Centering on Wilfred Carr's View of it [In Korean]. The Korean Journal of
Philosophy of Education, Vol. 45, 27- 51, 2009.

[8] MacIntyre, A. After Virtue: a Study in Moral Theory (2nd ed). London: Duckworth, 1984

[9] Lee, N.I. Phenomenology and Qualitative Research. [In Korean]. Seoul: HanGilSa, 2014.

[10] Peters, R.S. Ethics and Education. London: George Allen\& Unwin, 1966.

[11] Pring, R. Philosophy of Educational Research. London\& New York: Bloomsbury Publishing, 2015.

[12] Park, E. J. and Kwak, D. J. The Purpose and Method of Educational Studies revisited: Possibility for Practical Philosophy of Educational Studies [In Korean]. Korean Journal of Educational Research 54, 1-30, 2016.

[13] Yoo, J.B. A Conceptual Analysis of the MacIntyre's Conception of a Social Practice [In Korean]. The Korean Journal of Philosophy of Education, Vol. 24, 49-72, 2000 